\title{
A Tecnologia Assistiva e a Inclusão de Educandos com Deficiência Visual: um Mapeamento Sistemático da Literatura
}

\author{
Ana Carolina Candido de Melo1, Ellen Polliana Ramos Souza1, José Vinícius \\ Vieira Lima2 \\ 1Universidade Federal Rural de Pernambuco - (UFRPE) \\ 2Universidade de Pernambuco - (UPE) \\ \{carolina.melo, ellen.ramos\}@ufrpe.br, jvvl@ecomp.poli.br
}

\begin{abstract}
Assistive Technology (AT) refers to resources and services that extend the functional abilities of the Person with Disability $(P w D)$, aiming their social inclusion. Thus, this work presents the results of a systematic mapping of the literature, whose aim is to investigate the advances in the use of resources and services of AT for the inclusion of persons with visual impairment in the educational scope, through manual and automatic searches in the period from 2009 to 2018, proposing at the end, the cataloging and disclosure of these resources. A total of 12.279 primary studies were returned, which 30 were included in the mapping, among the results, highlighting educational software as the type of solution proposed.
\end{abstract}

Resumo. Tecnologia Assistiva (TA) refere-se a recursos e serviços que ampliam as habilidades funcionais da Pessoa com Deficiência (PcD), visando sua inclusão social. Assim, este trabalho apresenta o resultado de um mapeamento sistemático da literatura, cujo objetivo é investigar os avanços no uso de recursos e serviços de TA para inclusão da PcD Visual no âmbito educacional, através de buscas manual e automática no periodo de 2009 a 2018, propondo ao final, a catalogação e divulgação destes recursos. Foram retornados 12.279 estudos primários, dos quais, 30 foram incluídos no mapeamento. Dentre os resultados, destaque para softwares educativos como tipo de solução proposta.

\section{Introdução}

Os debates acerca da inclusão da Pessoa com Deficiência (PcD), em sua maioria, enfatizam o acesso desta comunidade a serviços essenciais, como exemplo a educação. Com isso, pesquisadores das áreas de educação e informática, têm buscado meios de oferecer recursos de apoio ao seu processo de ensino e aprendizagem. À PcD Visual, na maioria das vezes, é inexiste uma reflexão mais aprofundada sobre o dever institucional em contribuir para a sua inclusão educacional, sendo vários os obstáculos e problemas enfrentados para alcançar sua formação básica e superior [Schweitzer 2007].

De acordo com o último censo do Instituto Brasileiro de Geografia e Estatística (IBGE) em 2010, 18,6\% da população brasileira declararam ter deficiência visual, neste 
total estão incluídas aquelas com deficiência congênita ou adquirida, cegueira ou baixa visão. Especificamente, o letramento digital a deficientes visuais pode torná-los mais independentes, visando a busca de informações para desenvolvimento das mais diversas atividades, desde como resolver um problema matemático a como cozinhar determinado prato. Atrelado a isso, várias tecnologias vêm sendo aprimoradas para melhor atender esse público. Dentre essas tecnologias, estão os teclados adaptados com mecanismos em braille, softwares de leitura de telas, softwares que aumentam o tamanho de fontes e figuras, dentre outros [De Lima et al. 2018].

A Tecnologia Assistiva (TA) inclui auxílios técnicos personalizados para atender às necessidades da PcD. Bersch (2008) define TA como um termo utilizado para identificar o arsenal de recursos e serviços que contribuem para ampliar as habilidades funcionais da $\mathrm{PcD}$ e consequentemente promover vida independente e inclusão. Os recursos são itens ou sistemas fabricados e utilizados com o objetivo de melhorar as capacidades funcionais da PcD. Enquanto que, os serviços são definidos como aqueles que auxiliam diretamente a PcD a selecionar, adquirir ou utilizar os recursos de TA.

Em consequência disso, no universo da TA, o agrupamento dos estudos primários com foco no desenvolvimento de recursos para este segmento mostra-se crescente na academia, tendo em vista a quantidade de trabalhos disponíveis, tanto em plataformas de artigos nacionais quanto internacionais [Wohlin et al. 2013]. Contudo, para auxiliar no acesso dessas informações, existem os estudos secundários, que visam analisar estudos primários relativos a certas questões de pesquisa, promovendo aos pesquisadores uma visão geral de uma área de interesse [Kitchenham 2004].

Através de um Mapeamento Sistemático da Literatura dos últimos 10 anos, este trabalho tem como objetivo investigar os avanços no uso de recursos e serviços de TA para inclusão da PcD Visual no âmbito educacional, bem como disponibilizar um catálogo destes recursos em uma página WEB gratuita. O trabalho está estruturado da seguinte forma: a Seção 2 apresenta os trabalhos relacionados; $\mathrm{Na}$ Seção 3, são explanados os procedimentos metodológicos; A Seção 4 descreve os resultados; E, por fim, a Seção 5 apresenta as considerações finais e a continuidade deste trabalho.

\section{Trabalhos Relacionados}

Aguiar e Alves (2014) publicaram um estudo cujo objetivo foi a realização de um mapeamento sistemático a fim de descobrir como a TA e a acessibilidade estão sendo discutidas nos ambientes educacionais, considerando o período de 2010 a 2014 . Como principal resultado, em um total de 11 estudos primários considerados na pesquisa, foi apontada uma predominância de soluções para $\mathrm{PcD}$ visual em ambientes educacionais.

Neto (2015) realizou uma pesquisa bibliográfica manual nos anais do SBIE e WIE no período de 2001 a 2014, com o objetivo em identificar as soluções de TA que auxiliam o processo de inclusão da $\mathrm{PcD}$ nas universidades e mercado de trabalho. Ao final, 40 estudos primários foram considerados, e como resultado, identificou soluções em ferramentas e softwares educativos com foco na deficiência auditiva.

Freitas e Souza (2018), realizaram uma revisão sistemática da literatura, que investigou TAs para apoiar o ensino e aprendizagem na matemática para educandos com deficiência visual, no qual estudos primários de qualquer ano foram considerados. Os resultados apresentaram 59 tecnologias que possibilitam explorar diversos sentidos: tátil, auditivo, háptico e multimodal em diversos conteúdos da Matemática. 
Em contraste com os trabalhos relacionados, este trabalho realizou dois tipos de busca: manual e automática, por conseguinte foram consideradas mais fontes de pesquisa, bem como maior quantidade de questões de pesquisa. Posteriormente, realizou-se a catalogação dos recursos retornados da literatura, ilustrando a Tabela 1.

Tabela 1. Análise e comparação

\begin{tabular}{|c|c|c|c|}
\hline Autor(es) & Período & Busca & Fontes \\
\hline $\begin{array}{c}\text { Aguiar e Alves } \\
(2014)\end{array}$ & 2010 a 2014 & Automática & $\begin{array}{c}\text { ACM Digital Library - IEE Xplore } \\
\text { Digital Library - Scopus }\end{array}$ \\
\hline Neto (2015) & 2001 a 2014 & Manual & SBIE - WIE \\
\hline $\begin{array}{c}\text { Freitas e Souza } \\
(2018)\end{array}$ & Sem restrição & Automática & $\begin{array}{c}\text { IEE Xplore Digital Library - Scopus - } \\
\text { Web of Science }\end{array}$ \\
\hline Este Trabalho & $\mathbf{2 0 0 9}$ a 2018 & $\begin{array}{c}\text { Manual e } \\
\text { Automática }\end{array}$ & $\begin{array}{c}\text { SBIE - WIE - RBIE - RENOTE - } \\
\text { Portal do Períodicos CAPES - } \\
\text { SciELO - Google Scholar - SIBiUSP }\end{array}$ \\
\hline
\end{tabular}

\section{Método}

Neste trabalho, optou-se pela implementação de um mapeamento sistemático da literatura, sendo este um estudo secundário, com questões de caráter exploratório, permitindo reunir informações relevantes sobre uma área específica [Petersen et al. 2008]. O Método está organizado em cinco etapas, como mostra a Figura 1.

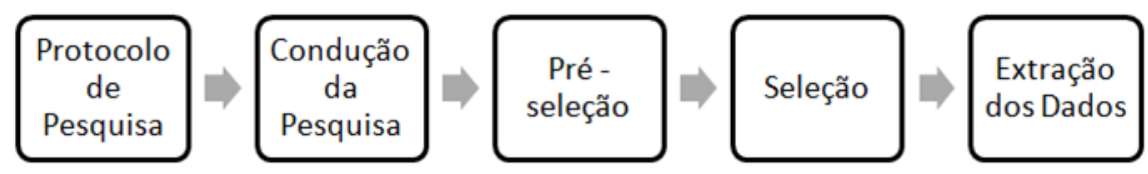

Figura 1. Etapas do mapeamento sistemático

Na primeira etapa, visando identificar e analisar evidências no uso de recursos e serviços de TA para inclusão da $\mathrm{PcD}$ visual no meio educacional, foram elaboradas às seguintes questões de pesquisa $(\mathrm{QP})$ :

QP1 - Qual a evolução do número de publicações de 2009 a 2018 ?

QP2 - Qual região do país tem predominância no estudo do tema?

QP3 - Quais os tipos de soluções propostas pelos autores nos estudos primários com o uso da tecnologia assistiva?

QP4 - Qual a distribuição dos estudos em relação ao nível de ensino? 
QP5- Quais benefícios e problemas foram observados com o uso das tecnologias assistivas na escola?

Em seguida, foram definidas as fontes e string de busca. Considerando a busca manual, optou-se por eventos e revistas de destaque na área de informática na educação. No processo da busca automática, as bibliotecas digitais foram escolhidas por possuírem relevância na academia, bem como disponibilidade para downloads de estudos primários, em especial estudos escritos em língua portuguesa, como mostra a Tabela 2.

Na Tabela 3, é apresentada a string de busca utilizada na busca automática. Nela, foram incluídos os termos "Tecnologia Assistiva" e "Educacional" com seus respectivos sinônimos para a seleção dos estudos primários.

Na sequência, foram definidos critérios de inclusão (CI) e de exclusão (CE) para garantir que apenas os estudos primários relevantes fossem considerados no mapeamento para responder às questões da pesquisa. Os critérios definidos foram:

CI-1 Estudos primários publicados entre 2009 e 2018;

CI-2 Estudos primários escritos em língua portuguesa;

CI-3 Estudos primários que apresentem o uso de tecnologia assistiva na inclusão educacional da pessoa com deficiência visual.

CE-1 O estudo primário não está disponível online;

CE-2 Estudos secundários (revisões e mapeamentos da literatura);

CE-3 O estudo primário é uma versão mais antiga de um outro estudo já considerado.

Tabela 2. Fontes de busca

\begin{tabular}{|l|l|}
\hline \multicolumn{1}{|c|}{ Manual } & \multicolumn{1}{c|}{ Automática } \\
\hline $\begin{array}{l}\text { Simpósio Brasileiro de Informática na Educação } \\
\text { (SBIE) }\end{array}$ & Portal de Períodicos CAPES \\
\hline Workshop de Informática na Escola (WIE) & Google Scholar \\
\hline $\begin{array}{l}\text { Revista Brasileira de Informática na Educação } \\
\text { (RBIE) }\end{array}$ & $\begin{array}{l}\text { Scientific Eletronic Library Online } \\
\text { (SciELO) }\end{array}$ \\
\hline Revista Novas Tecnologias na Educação (RENOTE) & Portal de Busca Integrada (SIBiUSP) \\
\hline
\end{tabular}

Tabela 3. String de busca

\section{String}

("tecnologia assistiva" OR "tecnologia acessível” OR "tecnologia de apoio" OR "ajuda técnica" OR "tecnologia de suporte" OR "tecnologia adaptativa")

AND

("educacional" OR "educativo" OR "educação" OR "escola" OR "ensino") 
Na segunda etapa, correspondente à condução da pesquisa, foi realizada a busca manual pelos estudos através do acesso manual as fontes de buscas. Na busca automática, os estudos foram identificados aplicando a string nas bibliotecas digitais.

A busca inicial resultou em 12.279 estudos primários, em seguida foram aplicados os critérios de inclusão e exclusão, resultando em um total de 480 estudos primários aprovados na etapa de pré-seleção, para serem selecionados nesta etapa, estes tiveram seus títulos, resumos e palavras-chave analisados, de modo a verificar sua conformidade aos critérios de inclusão definidos. Na etapa de seleção, todos os estudos primários incluídos nesta lista tiveram o seu conteúdo analisado na íntegra, de modo que nenhuma informação relevante fosse desconsiderada, como mostra a Tabela 4.

Tabela 4. Resultado da condução da pesquisa

\begin{tabular}{|c|c|c|c|c|c|c|c|c|c|}
\cline { 2 - 10 } \multicolumn{1}{c|}{} & \multicolumn{4}{c|}{ Busca Manual } & \multicolumn{4}{c|}{ Busca Automática } & \multirow{2}{*}{} \\
\cline { 2 - 10 } \multicolumn{1}{c|}{} & SBIE & WIE & RBIE & RENOTE & CAPES & GOOGLE & SCIELO & SIBiUSP & Total \\
\hline Buscas & 1.469 & 723 & 258 & 1.106 & 200 & 8.058 & 308 & 157 & 12.279 \\
\hline $\begin{array}{c}\text { Pré- } \\
\text { seleção }\end{array}$ & 121 & 54 & 21 & 57 & 46 & 77 & 61 & 43 & 480 \\
\hline Seleção & 34 & 13 & 3 & 20 & 9 & 21 & 3 & 1 & 104 \\
\hline
\end{tabular}

$\mathrm{Na}$ última etapa, correspondente a extração dos dados, buscou-se obter informações para responder as questões de pesquisa, sendo utilizada uma planilha eletrônica para organizar os dados. Ao final, 30 estudos primários voltados para a deficiência visual foram considerados para análise, estes disponíveis online.

\section{Resultados}

Nesta seção, são apresentadas as respostas para as questões de pesquisa.

\subsection{QP1 - Qual a evolução do número de publicações de 2009 a 2018 ?}

A Figura 2 ilustra os estudos analisados por ano de publicação, e evidencia que o número de publicações era crescente em anos anteriores, contudo, apresentou um declínio nos últimos anos. Destaque para o ano de 2010, com um total de 6 publicações.

\begin{tabular}{|c|l|}
\hline Ano & \multicolumn{1}{|c|}{ Relação de Estudos } \\
\hline 2009 & EP03, EP07,EP10 \\
\hline 2010 & EP11, EP13, EP16, EP17, EP18, EP21 \\
\hline 2011 & EP23, EP24, EP27 \\
\hline 2012 & EP29, EP30, EP35 \\
\hline 2013 & EP36, EP38, EP39, EP11, EP12 \\
\hline 2014 & EP49 \\
\hline 2015 & EP58, EP59, EP61, EP63 \\
\hline 2016 & EP66 \\
2017 & EP74, EP77 \\
\hline 2018 & EP84, EP88 \\
\hline
\end{tabular}

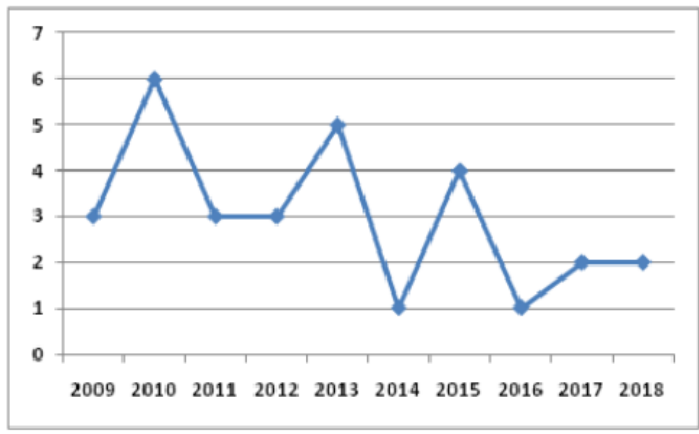

$1 \mathrm{http}: / /$ bit.ly/2UHSLNm 


\section{Figura 2. Evolução no número de publicações}

\subsection{QP2 - Qual região do país tem predominância no estudo do tema?}

Nesta questão foi identificado como se distribuiu o número de publicações por região do país. As regiões Sul e Sudeste se destacaram das demais, cada uma com um total de 11 publicações. A Tabela 5 apresenta estes e os demais resultados.

Tabela 5. Estudos por região do país

\begin{tabular}{|c|c|l|}
\hline Região & Quant. & \multicolumn{1}{c|}{ Relação de Estudos } \\
\hline Sul & 11 & EP03, EP10, EP13, EP27, EP29, EP30, EP35, EP39, EP49, EP77, EP84 \\
\hline Sudeste & 11 & EP07, EP11, EP16, EP17, EP18, EP24, EP36, EP58, EP59, EP61, EP63 \\
\hline Nordeste & 7 & EP21, EP23, EP38, EP41, EP66, EP74, EP88 \\
\hline Norte & 1 & EP42 \\
\hline
\end{tabular}

4.3. QP3 - Quais os tipos de soluções propostas pelos autores nos estudos primários com o uso da tecnologia assistiva?

Nota-se uma variedade de soluções a esta questão, agrupadas de acordo com a classificação definida pelo próprio autor do estudo, conforme mostra a Tabela 6 .

Tabela 6. Estudos por solução

\begin{tabular}{|c|c|l|}
\hline Solução & Quant. & \multicolumn{1}{|c|}{ Relação de Estudos } \\
\hline Software & 8 & EP03, EP07, EP21, EP29, EP30, EP49, EP63, EP88 \\
\hline Ferramenta & 6 & EP13, EP16, EP18, EP23, EP24, EP84 \\
\hline Aplicativo & 5 & EP17, EP39, EP59, EP66, EP77 \\
\hline Estudo & 5 & EP10, EP11, EP41, EP42, EP61 \\
\hline Ambiente de Aprendizagem & 4 & EP27, EP35, EP36, EP58 \\
\hline Objeto de Aprendizagem & 2 & EP74, EP78 \\
\hline
\end{tabular}

Ainda nesta questão, foi possível identificar o DOSVOX como o recurso de TA mais abordado entre os estudos, sendo este um software que se comunica com o usuário através de síntese de voz, viabilizando, o uso de computadores por deficientes visuais. Ademais, outros recursos foram citados, tal como o leitor de tela do Windows NVDA.

\subsection{QP4 - Qual a distribuição dos estudos em relação ao nível de ensino?}

Foi possível identificar que alguns estudos eram voltados para um nível de ensino em específico, com destaque para o Ensino Fundamental e Superior. A Tabela 7 mostra estes e os demais resultados.

Tabela 7. Estudos por nível de ensino

\begin{tabular}{|c|c|l|}
\hline Nível de Ensino & Quant. & Relação de Estudos \\
\hline Ensino Fundamental & 3 & EP10, EP11, EP21 \\
\hline
\end{tabular}




\begin{tabular}{|c|c|l|}
\hline Ensino Superior & 3 & EP16, EP41, EP42 \\
\hline Sala de Recurso & 2 & EP39, EP49 \\
\hline Ensino Técnico & 2 & EP13, EP16 \\
\hline Atendimento Educacional Especializado & 1 & EP77 \\
\hline
\end{tabular}

Nesta questão, foi possível identificar as disciplinas abordadas entre os estudos primários, como mostra a Tabela 8 . Apenas duas disciplinas foram identificadas.

Tabela 8. Estudos por disciplina

\begin{tabular}{|c|c|c|}
\hline Disciplina & Quant. & Relação de Estudos \\
\hline Informática & 6 & EP13, EP16, EP24, EP30, EP41, EP42 \\
\hline Matemática & 6 & EP17, EP36, EP38, EP49, EP74, EP84 \\
\hline
\end{tabular}

\subsection{QP5 - Quais benefícios e problemas foram observados com o uso das tecnologias assistivas na escola?}

Os relatos dos autores em alguns dos estudos primários analisados citam benefícios e problemas observados com o uso da TA em sala de aula, observações feitas pelo ponto de vista dos educadores e educandos com deficiência visual, como ilustra a Tabela 9.

Tabela 9. Benefícios e problemas ao utilizar a TA

\begin{tabular}{|c|l|}
\hline Estudos & \multicolumn{1}{c|}{ Benefícios } \\
\hline EP21, EP30, EP38 & $\begin{array}{l}\text { Dentre os resultados, identificou-se maior motivação por parte dos } \\
\text { alunos com deficiência em realizar as atividades. }\end{array}$ \\
\hline EP03 & $\begin{array}{l}\text { O uso em ambientes escolares segundo os depoimentos dos } \\
\text { professores, demonstra avanços no aproveitamento escolar. }\end{array}$ \\
\hline
\end{tabular}

\begin{tabular}{|c|l|}
\hline Estudos & \multicolumn{1}{c|}{ Problemas } \\
\hline EP11 & $\begin{array}{l}\text { Preocupação com a falta de responsabilidade dos jovens com os } \\
\text { próprios estudos, e na apropriação da tecnologia. }\end{array}$ \\
\hline
\end{tabular}

\subsection{Catálogo Online}

A partir do mapeamento sistemático, foi desenvolvido um catálogo online. O mesmo consiste em uma página WEB gratuita voltada para o meio educacional, com o objetivo em compartilhar recursos de TA para a PcD. Os recursos foram selecionados dentre aqueles retornados da literatura. Estes podem ser utilizados no processo de ensino e aprendizagem, fazendo com que a PcD realize suas atividades e aprenda de forma autônoma. Os recursos são organizados por deficiência, possuindo informações pedagógicas e técnicas. O link para acesso ao catálogo encontra-se disponível2. 
A Figura 3 ilustra na primeira imagem sua página inicial, e ao lado, a área onde é apresentado uma breve definição sobre a TA.
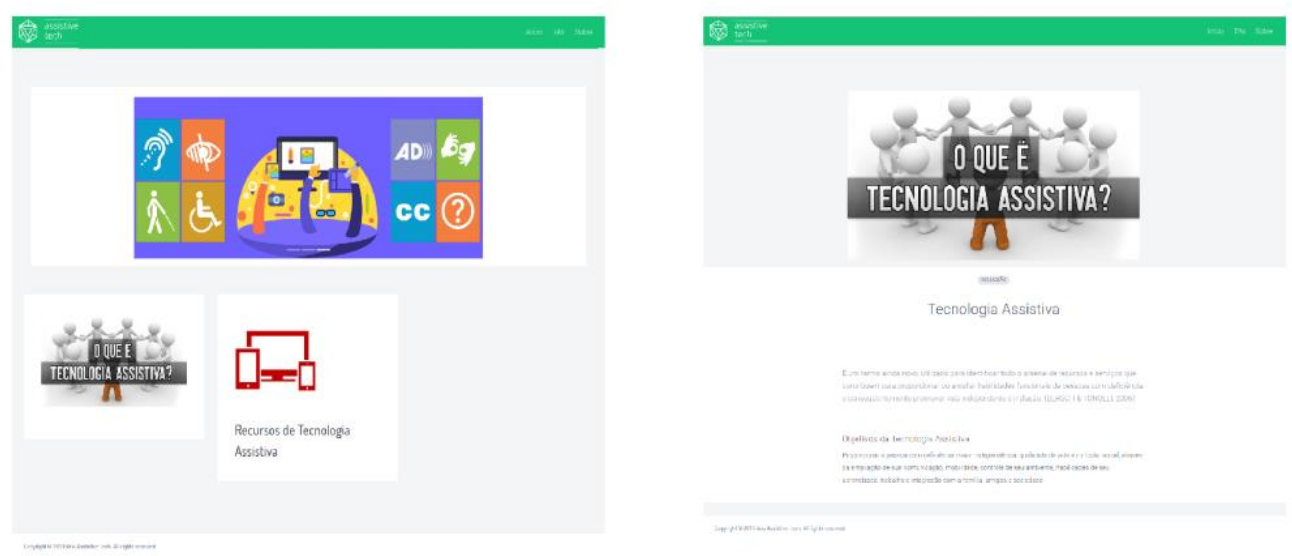

Figura 3. Páginas do catálogo

A Figura 4 apresenta a página que oferta os recursos de TA catalogados e disponíveis, estes estão organizados pelo seu nome e a deficiência que está voltado.

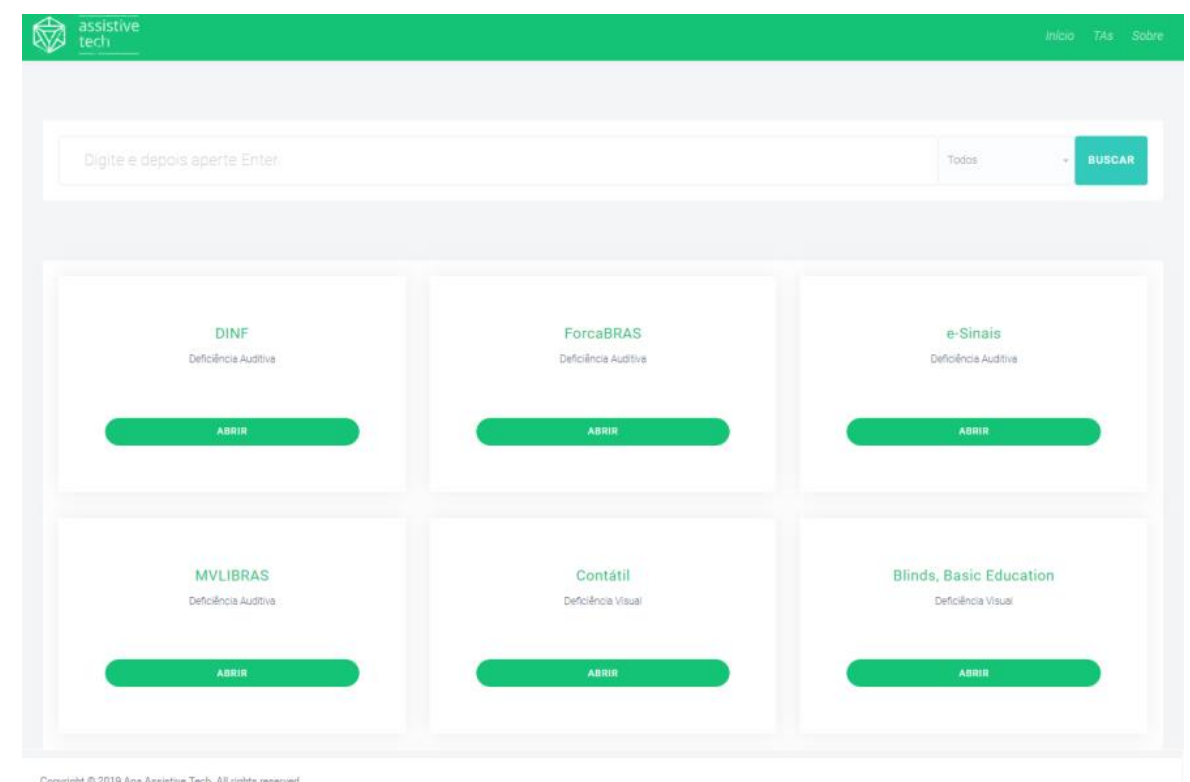

Figura 4. Página dos recursos

Ao selecionar um destes recursos, será aberta uma outra página contendo informações pedagógicas e técnicas, como mostra a Figura 5. 


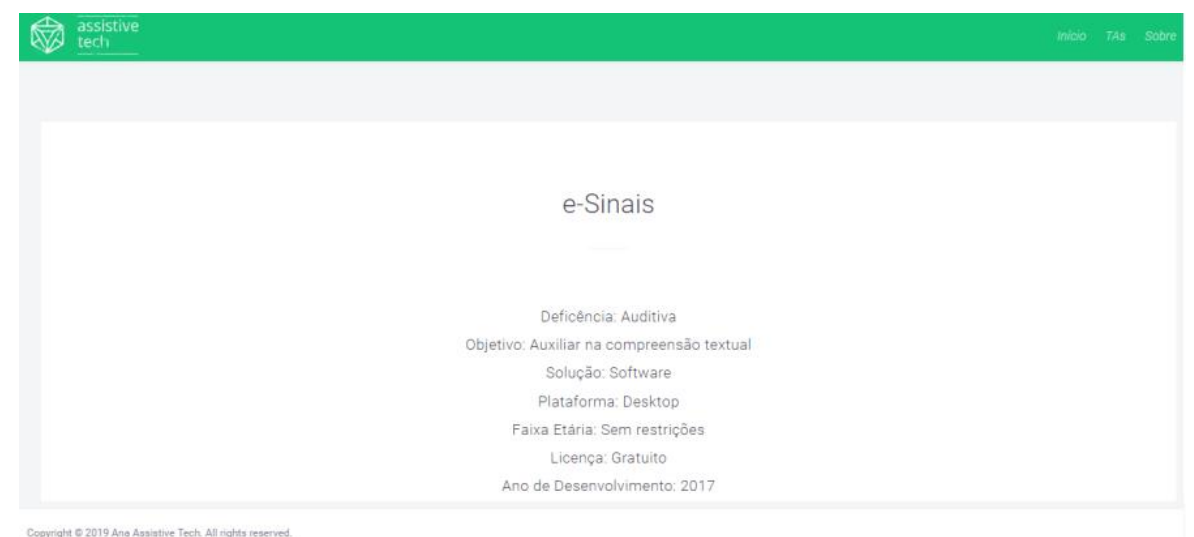

Figura 5. Informações dos recursos

\section{Considerações Finais e Trabalhos Futuros}

\subsection{Discussão dos Resultados}

Este mapeamento sistemático da literatura foi realizado tendo como base os 30 estudos primários voltados à deficiência visual. A partir da análise destes estudos, notou-se uma variação no número de publicações no período de 2009 a 2018, com destaque para o ano de 2010. Logo após, destaque para o estado do Rio Grande do Sul com mais publicações sobre o tema, um dos possíveis motivos é a UFRGS possuir um núcleo de pesquisa na área de educação inclusiva. Em seguida, um total de seis soluções propostas pelos autores foram identificadas, além dos níveis de ensino e disciplinas abordadas entre os estudos primários. Finalizando com os benefícios e problemas observados e relatados com o uso da TA em sala de aula.

Assim, este trabalho apresentou como principal contribuição uma visão geral, não enviesada e passível de replicação do estado da arte dos recursos digitais de TA desenvolvidos para auxiliar na inclusão educacional da $\mathrm{PcD}$, em especial aos educandos com deficiência visual. Neste sentido, seus resultados são úteis tanto para os educadores e PcD que necessitam fazer usos de tais recursos, como também para os pesquisadores que desejam investigar e desenvolver outros tipos de tecnologias no âmbito educacional.

O Catálogo desenvolvido com os recursos digitais de TA retornados da literatura, estes podem ser utilizados no processo de ensino e aprendizagem, fazendo com que a PcD realize suas atividades e aprenda de forma autônoma, estimulando suas habilidades e competências, proporcionando sua inclusão nos desafios em sala de aula. Ao final, se torna uma informação útil para quem está em busca de uma ferramenta acessível e que nem sempre tem conhecimento das ferramentas que já existem.

\subsection{Trabalhos Futuros}

A continuidade deste trabalho prevê a execução da mesma string de busca em outras bibliotecas digitais conceituadas para identificação de mais estudos primários e posteriormente de mais evidências que complementem os resultados obtidos até o momento. Além disso, essa atividade prevê a busca manual em outras conferências e revistas específicas da área, como exemplo o Congresso sobre Tecnologias na Educação (Ctrl+e), e o Workshop sobre Educação em Computação (WEI - CSBC). Também prevê a análise de estudos primários publicados em língua inglesa. 
Por fim, outras discussões, questões de pesquisa e deficiências deverão ser abordadas e investigadas para ampliar o entendimento do uso dos serviços e recursos digitais de TA para a inclusão educacional da $\mathrm{PcD}$, bem como a ampliação e divulgação do Catálogo desenvolvido, melhorando o mesmo nos aspectos de busca, organização e inserção de novas informações.

\section{Referências}

Alves, G. M. and Aguiar, Y. P. C. (2014). Acessibilidade e tecnologia assistiva no ambiente educacional: Mapeamento sistemático. In Anais do Workshop de Informática na Escola, volume 20, page 16.

Bersch, R. (2008). Introdução a tecnologia assistiva. Porto Alegre: CEDI, page 21.

De Lima, L. B. S., Dos Santos, L. C., and Moreira, K. C. (2018). O início de uma prática de letramento digital voltada para pessoas com deficiência visual. III Congresso sobre Tecnologias na Educação.

Kitchenham, B. (2004). Procedures for performing systematic reviews. Keele, UK, Keele University, 33(2004):1-26.

Neto, L. and de Souza, H. (2015). Abordagens para inclusão educacional e em ambientes de trabalho de pessoas com deficiência: um mapeamento sistemático em eventos brasileiros.

Petersen, K., Feldt, R., Mujtaba, S., and Mattsson, M. (2008). Systematic mapping studies in software engineering. In Ease, volume 8, pages 68-77.

Schweitzer, F. (2007). A sociedade e a informação para os deficientes visuais: relato de pesquisa the society and the information for to the deficient appearances: research report p. 273-285. Revista ACB, 12(2):273-285.

Souza, A. and Freitas, D. (2018). Tecnologias assistivas para apoiar o ensino e aprendizagem de pessoas com deficiência visual na matemática: Uma revisão sistemática da literatura. In Brazilian Symposium on Computers in Education (Simposio Brasileiro de Informatica na Educação-SBIE), volume 29, page 923.

Wohlin, C., Runeson, P., Neto, P. A. d. M. S., Engstrom, E., do Carmo Machado, I., and De Almeida, E. S. (2013). On the reliability of mapping studies in software engineering. Journal of Systems and Software, 86(10):2594-2610. 